\title{
Can diffusion weighted magnetic resonance imaging help differentiate stroke from stroke-like events in MELAS?
}

\author{
C Oppenheim^, D Galanaud ${ }^{\star}$, Y Samson, M Sahel, D Dormont, B Wechsler, C Marsault
}

\begin{abstract}
The precise mechanism of neurological symptoms in patients with mitochondrial myopathy, encephalopathy, lactic acidosis, and stroke-like episodes (MELAS) is still controversial. The diffusion weighted MR findings at the acute phase of a neurological event in MELAS are described and the pathophysiology of stroke-like lesion in the light of diffusion changes is discussed. Brain MRI was performed 2 days after the sudden onset of cortical blindness in a 25 year old patient with MELAS. Fluid attenuated inversion recovery (FLAIR) images showed multifocal cortical and subcortical hyperintensities located bilaterally in the frontobasal and the temporooccipital lobes. Diffusion weighted images showed normal to increased apparent diffusion coefficient values in the acute left temporooccipital lesion and increased values in the older stroke-like lesions.

These diffusion weighted findings support the metabolic rather than the ischaemic pathophysiological hypothesis for strokelike episodes occurring in MELAS. Normal or increased apparent diffusion coefficient values within 48 hours of a neurological deficit of abrupt onset should raise the possibility of MELAS, especially if conventional MR images show infarctlike lesions.

(F Neurol Neurosurg Psychiatry 2000;69:248-250)
\end{abstract}

Keywords: degenerative disease; diffusion weighted image ; mitochondrial encephalopathy

Among disorders resulting from impaired mitochondrial function, MELAS (mitochondrial encephalomyopathy, lactic acidosis, and strokelike events), first described in 1984 by Pavlakis et $a l,{ }^{1}$ is clinically characterised by nausea, vomiting, encephalopathy, seizures, short stature, headaches, diabetes mellitus, muscle weakness, exercise intolerance, neurosensory hearing loss, myopathy, and sudden neurological deficit. Although these neurological signs strongly mimic these of stroke, their exact mechanism is still unclear and thus, they are generally named "stroke-like" events. Two main hypotheses have been raised to explain brain involvement: angio- pathy leading to ischaemia or direct neuron death resulting from mitochondrial impairment. Conventional MRI shows signal abnormalities indistinguishable from those seen in ischaemic stroke. We describe the diffusion weighted MR pattern of an acute stroke-like event in an adult patient with MELAS established by biopsy and discuss the pathophysiology of stroke-like lesions in MELAS in the light of diffusion weighted findings.

\section{Case report}

A 25 year old patient of short stature $(1.59 \mathrm{~m})$ with a history of bilateral deafness and insulin dependent diabetes mellitus was admitted to hospital for a left sided hemianopsia of rapid onset with partial seizures. Conventional MRI performed at the acute phase of this first neurological event showed a large area of hypersignal on T2 weighted pulse sequences with associated brain swelling in the right temporo-occipital lobe. Cerebral angiography showed normal intracranial and extracranial vessels. A lumbar puncture showed increased lactates $(5.6 \mathrm{mmol} / 1$ (normal $<3 \mathrm{mmol} / \mathrm{l})$ ) in the CSF. The presence of ragged-red fibres on muscle biopsy raised the possibility of MELAS, which was confirmed by the presence of mutation at position 3243 in the mitochondrial genome. A treatment with carnithine, coenzyme $\mathrm{Q}$, and riboflavine was then initiated.

One year later, a second MRI was performed 48 hours (figure) after the onset of rapidly progressive aphasia and sudden cortical blindness. The following sequences were used: spin echo T1 weighted sagittal acquisition, fast spin echo T2 weighted axial acquisition, and fast fluid attenuated inversion recovery (FLAIR) acquisition. Diffusion weighted imaging with an echo planar spin echo pulse sequence was used with $6 \mathrm{~mm}$ slice thickness, $1.5 \mathrm{~mm}$ gap, 96×96 matrix, $28 \times 21 \mathrm{~cm}$ field of view, 4000/120 (repetition time (ms)/effective echo time (ms)). Five sets of images were successively acquired with five values of $b$, starting from $b=0 \mathrm{~s} / \mathrm{mm}^{2}$ and rising to $800 \mathrm{~s} / \mathrm{mm}^{2}$, with diffusion gradients applied in three orthogonal spatial directions. Dedicated software (Functool, General Electric) allowed calculation of the apparent diffusion coefficient (ADC). Regions of interest (ROIs) were positioned on diffusion 


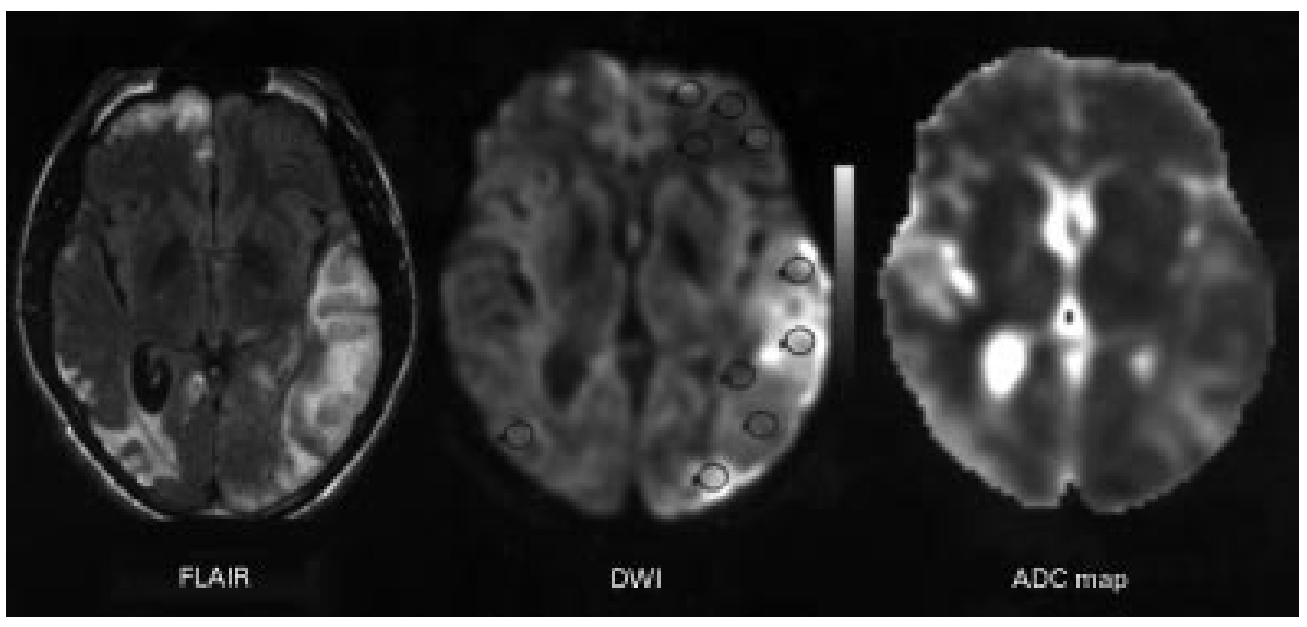

MRI performed 48 hours after the acute onset of a sudden cortical blindness. FLAIR image shows multifocal cortical and subcortical hyperintensities located in the right frontal lobe, and, bilaterally in the temporo-occipital lobes. Gyral swelling with mass effect on the adjacent ventricular horn can be seen in the acute left temporo-occipital lesion. The right temporo-occipital lesion is a sequal to the first neurological event that occurred a year ago. Although these multifocal signal abnormalities strongly mimic that of stroke (cortical swelling, sharp limits), the lesions have no vascular distribution. Diffusion weighted pulse sequence shows a heterogeneous area of iso/hypersignal in the left temporo-occipital area. No signal abnormality is seen within the right temporo-occipital sequel lesion ADC map. With the grey coloured scale used, increased $A D C$ values are displayed in white, decreased ADC values in black. The ROIs are positioned on diffusion weighted images and the mean $A D C$ values are given in table 1. ADC values are normal to increased in the acute left temporo-occipital lesion. Higher ADC values are seen in the right temporo-occipital chronic lesion.

weighted images in cortical, subcortical, and white matter areas alternatively in normal and abnormal appearing areas. An MR angiography of the circle of Willis and its major branches was also obtained and displayed normal vessels.

FLAIR sequences showed multifocal areas of high signal in the right frontobasal lobe and in the temporo-occipital lobes bilaterally. When compared with the MRI performed a year earlier (not shown), an additional lesion was seen in the left temporo-occipital lobe, matching the acute clinical presentation. On FLAIR images, this acute left temporooccipital lesion showed striking hypersignal with swelling gyri and mass effect. In this region, diffusion weighted images showed a heterogeneous signal with areas of isosignal and hypersignal. The ADC values calculated in the left temporo-occipital region were normal or increased when compared with the normal appearing left frontal corticosubcortical region. Diffusion weighted images showed no signal changes in the right sequellar temporooccipital lesion and $\mathrm{ADC}$ values were increased. Detailed mean ADC values are listed on the table.

Table 1 Mean apparent diffusion coefficient (ADC) values in regions of interest (ROIs) positioned on diffusion weighted images in normal and abnormal appearing areas

\begin{tabular}{lll}
\hline & $R O I$ & $\begin{array}{l}\text { Mean } \mathrm{ADC} \text { value } \\
\left(10^{-3} \mathrm{~mm}^{2} / \mathrm{s}\right)\end{array}$ \\
\hline Normal appearing grey matter (left frontal lobe) & 1 & 0.91 \\
& 2 & 0.97 \\
Normal appearing white matter (left frontal lobe) & 3 & 1.01 \\
Lesion at the chronic stage (right temporo-occipital cortex) & 4 & 0.83 \\
Cortico/subcortical acute lesion (left temporo-occipital lesion) & 5 & 1.70 \\
& 7 & 1.11 \\
& 8 & 0.68 \\
Abnormal deep white matter (adjacent to the left atrium) & 9 & 1.30 \\
& 10 & 1.30 \\
\hline
\end{tabular}

A statistical analysis using an unpaired two tailed Student's $t$ test showed a significant difference $(\mathrm{p}=0.03)$ between ADC values in ROIs positioned in normal appearing brain regions (mean 0.93 $(\mathrm{SD} 0.08) \mathrm{mm}^{2} / \mathrm{s}$ ) and those located in acute lesions (mean $1.26(\mathrm{SD} 0.24) \mathrm{mm}^{2} / \mathrm{s}$ ).
The condition of the patient rapidly deteriorated and he died the following month. Permission for necropsy was not granted.

\section{Discussion}

The focal neurological deficits of abrupt onset landmarking the evolution of MELAS are clinically indistinguishable from stroke events. ${ }^{2}$ Brain MRI of patients with MELAS classically shows signal changes involving both grey and white matter predominantly in the occipital and parietal lobes ${ }^{3}$ that strongly mimic stroke lesions. However, distribution of these infarctlike lesions on MRI does not usually follow vascular territories and pathological studies do not find lesions of the major cerebral blood vessels. Thus, the physiopathology of this disease remains unclear. Two main hypotheses have been raised to explain these cerebral lesions.

(1) The vascular hypothesis: metabolic damage of the endothelium leads to small vessel occlusion and secondary neuronal death. It is supported by the clinical course of the disease similar to that of stroke and the CT and MRI appearance of the lesions and some pathological reports of endothelium alterations in the brain of patients with MELAS. ${ }^{4}$

(2) The defect in neuronal metabolism hypothesis: mitochondrial dysfunction results in anaerobic metabolism and neuronal death from acidosis. It relies on PET and SPECT studies showing hyperperfusion and dissociation between glucose and oxygen consumption in the affected areas of the brain. ${ }^{5}$

Diffusion weighted imaging is a new technique in which images display local movement possibilities of water molecules ${ }^{67}$ that can help distinguish between cellular and extracellular oedema. ${ }^{8}$ Indeed, experimental results and human studies suggest that decreased ADC values reflects cellular oedema, ${ }^{9}$ whereas in- 
creased ADC values correspond to increased of the extracellular space-that is, extracellular oedema. ${ }^{1011}$ It has been well documented that cellular oedema during acute infarction is characterised by markedly decreased diffusion. It has been shown that ADC starts diminishing very early in the course of acute human stroke, reaches a minimum 2 or 3 days after onset, ${ }^{12}$ and remains under normal values for about 144 hours. ${ }^{13}$ It is thus now recognised that the finding of normal or increased ADC values within a few days after a neurological deficit of sudden onset virtually rules out acute ischaemic stroke. ${ }^{14}$ Although the duration of insult remains unknown in MELAS, the neurological symptoms of this patient were of sudden onset. Brain MRI was obtained 48 hours after this sudden onset and displayed normal or increased ADC values in brain areas undergoing acute dysfunction. Although a very short lasting ADC decrease immediately followed by an increase of ADC values cannot be excluded, such a rapid time course has not yet been reported in large acute arterial stroke in humans. ${ }^{12}{ }^{13}$ The ADC pattern we found 48 hours after onset strongly differs from the marked ADC decrease that should be seen at this time point in pure acute arterial stroke and thus does not favour ischaemic damage as the main mechanism explaining focal neurological deficit in MELAS. This confirms the recent finding of increased ADC values at the acute phase of a small stroke-like lesion in a case of childhood onset MELAS ${ }^{15}$ and extends this result to a large stroke-like lesion in MELAS of adult onset.

\section{Conclusion}

Unlike routine $\mathrm{MR}$ imaging, diffusion weighted imaging can discriminate between cellular and extracellular oedema. By showing increased $\mathrm{ADC}$ values consistent with predominant extracellular oedema in an acute lesion in MELAS, diffusion weighted imaging adds further evidence against an ischaemic mechanism for stroke-like events in MELAS. The finding of normal to increased ADC values within 48 hours of a neurological deficit of abrupt onset should raise the possibility of MELAS, especially if conventional MR shows infarct-like lesions.

1 Pavlakis SG, Phillips PC, DiMauro S, et al. Mitochondrial myopathy, encephalopathy, lactic acidosis and strokelike episodes: a distinctive clinical syndrome. Ann Neurol 1984;16:481-8.

2 Castillo M, Kwock L, Green C. MELAS syndrome: imaging and proton MR spectroscopic findings. AfNR Am f Neuroradiol 1995;16:233-9.

3 Barkovich AJ, Good WV, Koch TK, et al. Mitochondrial disorders: analysis of their clinical and imaging characteristics. AfNR Am f Neuroradiol 1993;14:1119-374.

4 Ohama E, Ohara S, Ikuta F, et al. Mitochondrial angiopathy in cerebral blood vessels of mitochondrial encephalomyopathy. Acta Neuropathol 1987;74:226-33.

5 Clark JM, Marks MP, Adalsteinsson E, et al. MELAS: clinical and pathologic correlations with MRI, Xenon/CT, and MR spectroscopy. Neurology 1996;46:223-7.

6 Le Bihan D, Breton E, Lallemand D, et al. MR imaging of intravoxel incoherent motions: application to diffusion and perfusion in neurologic disorders. Radiology 1986;161:401perfusi

7 Moseley ME, Cohen Y, Mintorovitch J, et al. Early detection of regional cerebral ischemia in cats: comparison of diffusion- and T2-weighted MRI and spectroscopy. Magn Reson Med 1990;14:330-46.

8 Schaefer PW, Buonanno FS, Gonzalez RG, et al. Diffusion weighted imaging discriminates between cytotoxic and vasogenic edema in a patient with eclampsia. Stroke 1997; 28:1082-5.

9 Loubinoux I, Volk A, Borredon J, et al. Spreading of vasogenic edema and cytotoxic edema assessed by quantitative diffusion and T2 magnetic resonance imaging. Stroke 1997;28:419-26.

10 Kuroiwa T, Nagaoka T, Ueki $M$, et al. Different apparent diffusion coefficient: water content correlations of gray and diffusion coefficient: water content correlations of gray and
white matter during early ischemia. Stroke 1998;29:85965.

11 Ito J, Marmarou A, Barzo P, et al. Characterization of edema by diffusion-weighted imaging in experimental traumatic brain injury. I Neurosurg 1996;84:97-103.

12 Schlaug G, Siewert B, Benfield A, et al. Time course of apparent diffusion coefficient (ADC) abnormality in human stroke. Neurology 1997;49:113-19.

13 Schwamm LH, Koroshetz WJ, Sorensen G, et al. Time course of lesion development in patients with acute stroke. Serial diffusion and hemodynamic weighted magnetic resonance imaging. Stroke 1998;29:2268-76.

14 Warach S, Gaa J, Siewert B, et al. Acute human stroke studied by whole brain echo planar diffusion weighted magnetic resonance imaging. Ann Neurol 1995;37:231-41.

15 Yoneda M, Maeda M, Kimura H, et al. Vasogenic edema on MELAS: a serial study with diffusion-weighted MR imaging. Neurology 1999;53:2182-4. 